\title{
Biologic therapy for systemic juvenile idiopathic arthritis -times they are a'changing!
}

$\mathrm{S}$ ystemic juvenile idiopathic arthritis (sJIA) has, until recently, been considered a therapeutic orphan. High-dose glucocorticoids, which are associated with serious toxicities, are the mainstay of treatment for chronic childhood polyarthritides. Two papers now published in The New England Journal of Medicine confirm, however, that changing times are ahead in sJIA therapy.

IL- 6 and IL- 1 have key roles in the pathogenesis of sJIA. So, members of the PRINTO and PRCSG networks decided to embark on phase III clinical trials of monoclonal antibodies that block these cytokines in patients with sJIA.

The first paper, by De Benedetti et al., reports data from a trial assessing the safety and efficacy of tocilizumab (a humanized anti-IL-6R monoclonal antibody) versus placebo in patients with persistent sJIA (according to ILAR criteria), with or without fever, who had inadequate responses to NSAIDs and glucocorticoids. 112 children were randomly assigned to tocilizumab ( $n=75 ; 8 \mathrm{mg} / \mathrm{kg}$ body weight if $\geq 30 \mathrm{~kg} ; 12 \mathrm{mg} / \mathrm{kg}$ body weight if $<30 \mathrm{~kg}$ ) or placebo $(n=37)$ intravenously every 2 weeks for 12 weeks (double-blinded phase). A 5-year open-label extension study was also performed.

At week $12,85 \%$ of patients treated with tocilizumab versus $24 \%$ of patients in the placebo group achieved the primary outcome of an adapted JIA ACR30 response (ACR criteria for 30\% improvement in JIA: absence of fever and improvement in $\geq 30 \%$ in three or more of the six variables of the JIA core set, with no more than one variable worsening by $>30 \%)$. In the open-label extension phase at week $52(n=110,73$ who had previously been assigned to receive tocilizumab and 37 who had previously been assigned to placebo), $80 \%$ of patients receiving tocilizumab achieved a JIA ACR70 response; $52 \%$ were able to discontinue glucocorticoids. In the double-blinded and

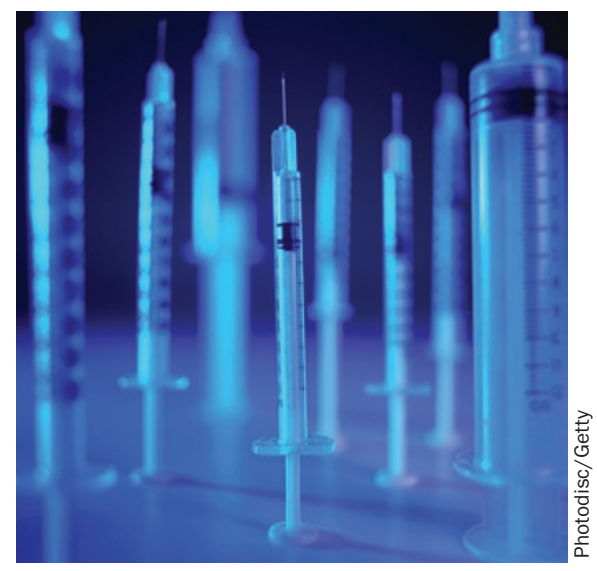

extension phases combined, in patients receiving tocilizumab 39 serious adverse events (including 18 serious infections) occurred and neutropenia developed in 19 patients; 3 patients died during the conduct of the study and 3 patients died after tocilizumab discontinuation.

The paper by Ruperto et al. reports findings from two trials assessing the safety and efficacy of canakinumab (a fully human anti-IL-1 $\beta$ monoclonal antibody) versus placebo in patients with active sJIA (defined as fever, active disease in $\geq 2$ joints, C-reactive protein level $>30 \mathrm{mg} / \mathrm{l}$, glucocorticoid dose $\leq 1 \mathrm{mg} / \mathrm{kg}$ body weight per day). In trial 1 , children were randomly assigned to a single subcutaneous dose of canakinumab ( $n=43 ; 4 \mathrm{mg} / \mathrm{kg}$ body weight) or placebo $(n=41)$ : a JIA ACR30 response was achieved in $84 \%$ of patients receiving canakinumab versus $10 \%$ of patients in the placebo group.

In trial 2, all patients were given canakinumab every 4 weeks for up to 32 weeks depending on steroid tapering schedule; those who achieved at least an adapted JIA ACR50 response and who were receiving glucocorticoids underwent glucocorticoid therapy tapering from week 9 (open-label phase). Then, patients who had achieved an adapted JIA ACR50 response and who were not receiving glucocorticoids (or who had undergone successful glucocorticoid tapering) were randomly assigned to either canakinumab or placebo (double-blind withdrawal phase). At the end of the open-label phase of trial 2,73\% of patients had received at least a JIA ACR50 response and 31\% had inactive disease. In the withdrawal phase, $74 \%$ of patients receiving canakinumb had no disease flare versus $25 \%$ in the placebo group. $33 \%$ of patients who were receiving glucocorticoids at study entry were able to discontinue these agents. Infections and macrophage activation syndrome (MAS) were more frequent in patients receiving canakinumab rather than placebo; two patients receiving canakinumab died during trial 2 and two patients died after canakinumab discontinuation.

"These studies are proof of the remarkable response to IL-1 and IL-6 inhibition in sJIA patients," says Yukiko Kimura (Joseph M. Sanzari Children's Hospital at Hackensack University Medical Center, USA) who was an investigator in the tocilizumab trial. "However, there are concerns regarding the safety of these agents", she concludes.

Similar conclusions were drawn by Norman Ilowite (Albert Einstein College of Medicine, USA), who was not involved in these studies."Both agents appear to enable successful tapering of corticosteroids, which is of great importance given the toxicities of corticosteroids in children." He adds however that, "more robust safety data in large numbers of exposed and nonexposed sJIA patients over long periods of time should be generated to better understand the significance of rare events such as MAS in treated patients."

Jenny Buckland

\footnotetext{
Original articles De Benedetti, F. et al. Randomized trial of tocilizumab in systemic juvenile idiopathic arthritis. N. Engl. J. Med. 367, 2385-2395 (2012) | Ruperto, N. et al. Two randomized trials of canakinumab in systemic juvenile idiopathic arthritis. N. Engl. J. Med. 367, 2396-2406 (2012)
} 